BULLETIN OF THE

AMERICAN MATHEMATICAL SOCIETY

Volume 77, Number 3, May 1971

\title{
BIHARMONIC CLASSIFICATION OF RIEMANNIAN MANIFOLDS ${ }^{1}$
}

\author{
BY M. NAKAI AND L. SARIO
}

Communicated by W. Fuchs, October 26, 1970

We consider a noncompact orientable $C^{\infty}$ manifold $R$ of dimension $m \geqq 2$ with a $C^{\infty}$ Riemannian metric $d s^{2}=\sum_{i, j=1}^{m} g_{i j} d x^{i} d x^{j}$. The corresponding Laplace-Beltrami operator is

$$
\Delta \cdot=-g^{-1 / 2} \sum_{i=1}^{m} \frac{\partial}{\partial x^{i}}\left(\sum_{j=1}^{m} g^{1 / 2} g^{i j} \frac{\partial \cdot}{\partial x^{j}}\right),
$$

with $g=\operatorname{det}\left(g_{i j}\right)$ and $\left(g^{i j}\right)=\left(g_{i j}\right)^{-1}$. Let $\varphi$ and $\psi$ be continuous functions on the Wiener or Royden boundary $\beta$ of $R$ (cf. [2] and [5]; the terminology used in the present note is adopted from [5]). We are interested in solving the biharmonic boundary value problem

$$
\Delta^{2} u=0, \quad u|\beta=\varphi, \quad \Delta u| \beta=\psi .
$$

Here $u$ and $\Delta u$ are required to be continuously extendable to the Wiener or Royden compactification $R^{*}$ of $R$. If $\varphi=\psi=0$ on the harmonic boundary $\delta \subset \beta$, then the solution of (2) vanishes identically. The problem, therefore, is to solve

$$
\Delta^{2} u=0, \quad u|\delta=\varphi, \quad \Delta u| \delta=\psi .
$$

Such problems are of fundamental importance in applications of biharmonic functions to physics, in particular the theory of bending of thin plates (cf. [1]). Unconditional solvability cannot be expected, since the solution of (3) clearly exists for the unit ball $R$ in the Euclidean space $E^{m}$ but not for $R=E^{m}$. This simple example illustrates the significance of the biharmonic classification problem in the theory of elasticity. Here we shall announce some results on the problem; the proofs will appear elsewhere (cf. [3], [4]).

1. Quasiharmonic functions. The simplest nonharmonic biharmonic functions are what we shall call quasiharmonic functions $u$, characterized by $\Delta u=$ const $\neq 0$. The normalized class $Q=Q(R)$ $=\left\{u \in C^{2}(R) \mid \Delta u=1\right\}$ can be viewed as a special class of super-

AMS 1969 subject classifications. Primary 3130, 3045.

Key words and phrases. Biharmonic classification, biharmonic measure, biharmonic projection, quasiharmonic function, Riesz representation.

1 The work was sponsored by the U. S. Army Research Office-Durham, Grant DA-ARO-D-31-124-70-G7, University of California, Los Angeles. 
harmonic functions on $R$. Let $P, B$, and $D$ stand for the classes of positive, bounded, and Dirichlet finite functions, and set $B D=B \cap D$. Consider the class $O_{Q X}$ of Riemannian ma iifolds $R$ on which $Q X(R)$ $=\varnothing$. Denote by $G(x, y)$ the harmonic Green's function on $R \notin O_{G}$ and set $G(x, y)=\infty$ on $R \in O_{G}$. We have the following complete characterizations:

$$
\begin{aligned}
& R \in O_{Q P} \text { if and only if } a=\int_{R} G(x, y) d y=\infty ; \\
& R \in O_{Q B} \text { if and only if } b=\sup _{x \in R} \int_{R} G(x, y) d y=\infty ; \\
& R \in O_{Q D} \text { if and only if } c=\int_{R \times R} G(x, y) d x d y=\infty ; \\
& R \in O_{Q B D} \text { if and only if } b=\infty \text { or } c=\infty .
\end{aligned}
$$

Here $d x=g^{1 / 2} d x^{1} \cdots d x^{m}$ is the Riemannian volume element on $R$.

By means of these characterizations we also obtain the inclusion relations

$$
O_{G} \rightarrow O_{Q P} \overbrace{O_{Q D}}^{O_{Q B}} \overbrace{O_{Q B D} .}
$$

Here $X \rightarrow Y$ stands for $X \underset{\not}{\subsetneq} Y$, and $X \backsim Y$ means that $X \nsubseteq Y$ and $Y \nsubseteq X$.

2. Riesz representation. For a class $X$ of functions and an operation $T$ on certain functions we denote by $X_{T}$ the class $\{f \mid T f$ is defined and $T f \in X\}$. Let $W=W(R)$ be the class of biharmonic functions $u$ on $R$, defined by $\Delta^{2} u=0$ with $u \in C^{4}(R)$. Suppose $u \in W X P_{\Delta}(R)$ with $X=P, B$, or $D$. We can establish the Riesz representation

$$
u(x)=H_{1} u(x)+\int_{R} G(x, y) H_{2} u(y) d y,
$$

with $H_{1} u \in H X(R)$ and $H_{2} u=\Delta u \in H P(R)$. The decomposition is unique. We call the function $H_{1} u$ the harmonic part of $u$, and the function $\mathrm{GH}_{2} u$ the potential part of $u$, with

$$
(G f)(x)=\int_{R} G(x, y) f(y) d y .
$$

Here $u, H_{i} u(i=1,2)$, and $G H_{2} u$ are continuous on the Wiener com- 
pactification $R^{*}=R_{N}^{*}$ of $R$, and $u=H_{1} u$ or equivalently $G H_{2} u=0$ on the Wiener harmonic boundary $\delta=\delta_{N}$. In the case $X=D$, we can replace $R_{N}^{*}$ and $\delta_{N}$ by the Royden compactification $R_{M}^{*}$ and the Royden harmonic boundary $\delta_{M}$, except regarding $H_{2} u$, and obtain

$$
D_{R}(u)=D_{R}\left(H_{1} u\right)+\int_{R \times R} G(x, y) H_{1} u(x) H_{2} u(y) d x d y .
$$

The mapping $\gamma u=\left(H_{1} u, H_{2} u\right)$ is an injective linear transformation from $W X P_{\Delta}$ into $H X \times H P$. To describe the image of $\gamma$, let $F=\{f \mid f$ is measurable $\}$ and $F_{0}=\{f \in F|G| f \mid(x)<\infty\}$. Then clearly

$$
\gamma\left(W X P_{\Delta}\right)=H X \times H P F_{0} .
$$

3. Classes of biharmonic functions. For the sake of brevity we also use the notation $C$ for $B D$ whenever it is obvious that we are not referring to the class of continuous functions. The solvability of (3) depends on the existence of the representation (6) and the surjectivity of $\gamma$ for the class $W B B_{\Delta}$ and its subclasses $W B C_{\Delta}, W C B_{\Delta}$, and $W C C_{\Delta}$. In view of

$$
O_{G} \subsetneq O_{W B B_{\Delta}},
$$

we may suppose that $M \notin O_{G}$. As a consequence of (6) we conclude:

If $R \notin O_{Q P}$, then the representation (6) is valid for $u \in W B B_{\Delta}$.

We stress that in (6) for $u \in W B B_{\Delta}$ we have the requirement

$$
\int_{R} G(x, y)\left|H_{2} u(y)\right| d y<\infty .
$$

The unrestricted solvability of (3) is then equivalent to the surjectivity of $\gamma$, which is characterized as follows:

$$
\begin{aligned}
& \gamma\left(W B B_{\Delta}\right)=H B \times H B \text { if and only if } R \in O_{Q B} ; \\
& \gamma\left(W B C_{\Delta}\right)=H B \times H C \text { if and only if } R \notin O_{Q B} ; \\
& \gamma\left(W C B_{\Delta}\right)=H C \times H B \text { if and only if } R \notin O_{Q C} ; \\
& \gamma\left(W C C_{\Delta}\right)=H C \times H C \text { if and only if } R \notin O_{Q C} .
\end{aligned}
$$

4. Biharmonic projection. The boundary functions in (3) are restrictions to $\delta$ of functions in the Wiener algebra $N$ or the Royden algebra $M$ on $R$. The following decomposition suggests another approach to solving (3). Let $F_{1}=\left\{f \in F_{0}\left|\sup _{x \in M}\right| G f(x) \mid<\infty\right\}$ and $F_{2}=\{f \in F \mid G(|f|,|f|)<\infty\}$. Here

$$
G(f, g)=\int_{R \times R} G(x, y) f(x) g(y) d x d y,
$$


which already occurred in (8), is referred to as the mutual energy of $f$ and $g$. If $f_{i} \in F_{2}$ and $G f_{i} \in C^{2}(R)(i=1,2)$, then

$$
D_{R}\left(G f_{1}, G f_{2}\right)=G\left(f_{1}, f_{2}\right) \text {. }
$$

Let

$$
\begin{aligned}
& \Phi_{1}=N N_{\Delta}\left(F_{1}\right)_{\Delta}\left(F_{1}\right)_{\pi \Delta}, \quad \Phi_{2}=N M_{\Delta}\left(F_{1}\right)_{\Delta}\left(F_{1}\right)_{\pi \Delta}, \\
& \Phi_{3}=M N_{\Delta}\left(F_{1}\right)_{\Delta}\left(F_{1}\right)_{\pi \Delta}\left(F_{2}\right)_{\Delta}\left(F_{2}\right)_{\pi \Delta}, \\
& \Phi_{4}=M M_{\Delta}\left(F_{1}\right)_{\Delta}\left(F_{1}\right)_{\pi \Delta}\left(F_{2}\right)_{\Delta}\left(F_{2}\right)_{\pi \Delta},
\end{aligned}
$$

where $\pi$ is the harmonic projection on $N$ or $M$. We denote by $N_{\delta}$ and $M_{\delta}$ the potential subalgebras of $N$ and $M$, defined by $N_{\delta} \mid \delta=0$ and $M_{\delta} \mid \delta=0$. The following direct decompositions are valid:

$$
\begin{array}{ll}
\Phi_{1}=W \Phi_{1}+\left(N_{\delta} \Phi_{1}\right)\left(N_{\delta}\right)_{\Delta} ; & \Phi_{2}=W \Phi_{2}+\left(N_{\delta} \Phi_{2}\right)\left(M_{\delta}\right)_{\Delta} ; \\
\Phi_{3}=W \Phi_{3}+\left(M_{\delta} \Phi_{3}\right)\left(N_{\delta}\right)_{\Delta} ; & \Phi_{4}=W \Phi_{4}+\left(M_{\delta} \Phi_{4}\right)\left(M_{\delta}\right)_{\Delta} .
\end{array}
$$

We shall call the projection $\tau_{i} f$ of $f \in \Phi_{i}$ to $W \Phi_{i}$ the biharmonic projection. It is given by

$$
\begin{aligned}
\tau_{i} f & =\pi f+G \pi \Delta f, \\
f-\tau_{i} f & =G(\Delta f-\pi \Delta f) .
\end{aligned}
$$

Here $W \Phi_{1} \subset W B B_{\Delta}, W \Phi_{2} \subset W B C_{\Delta}, W \Phi_{3} \subset W C B_{\Delta}$, and $W \Phi_{4} \subset W C C_{\Delta}$. The inclusions are replaced by equalities if $R \notin O_{Q B}, R \notin O_{Q B}, R \notin O_{Q C}$, and $R \notin O_{Q C}$, respectively; in these cases $\Phi_{1}=N N_{\Delta}, \Phi_{2}=N M_{\Delta}$, $\Phi_{3}=M N_{\Delta}$, and $\Phi_{4}=M M_{\Delta}$.

5. Biharmonic measure. Let $P(x, \zeta)$ with $(x, \zeta) \in R \times \beta$ be the harmonic kernel. We shall refer to the function

$$
K(x, \zeta)=\int_{R} G(x, y) P(y, \zeta) d y,
$$

which may or may not be finite, as the biharmonic kernel. If it is finite, then

$$
\Delta_{x} K(x, \zeta)=P(x, \zeta) .
$$

Suppose $\varphi \in H B|\delta=N| \delta$ and $\psi \in H B F_{1}\left|\delta=N F_{1}\right| \delta$. Then the solution of (3) is given by

$$
u(x)=\int_{\delta}(P(x, \zeta) \varphi(\zeta)+K(x, \zeta) \psi(\zeta)) d \mu(\zeta),
$$

where $\mu$ is the harmonic measure on $\delta$. If $\varphi$ and $\psi$ are $\mu$-measurable on 
$\delta$ and the integral in (17) is well defined, then the function (17) is biharmonic on $R$. If, moreover, $\varphi$ (resp. $\psi$ ) is continuous at some $\zeta \in \delta$, then $u$ (resp. $\Delta u$ ) has the continuous boundary value $\varphi(\zeta)$ (resp. $\psi(\zeta)$ ) at $\zeta$.

We define the biharmonic measure of a Borel set $E \subset \beta$ relative to $x \in R$ as

$$
\kappa_{x}(E)=\int_{E} K(x, \zeta) d \mu(\zeta)
$$

and the absolute biharmonic measure as

$$
\kappa(E)=\sup _{x \in M} \kappa_{x}(E) .
$$

The latter is not a genuine measure but an outer measure on $\beta$. We call a point $\zeta \in \beta$ absolutely singular if $\kappa(U)=\infty$ for every neighborhood $U$ of $\zeta$ in $\beta$. The set $S$ of such points constitutes a compact subset of $\delta$. We consider the function

$$
l_{E}(x)=\int_{E} P(x, \zeta) d \mu(\zeta)
$$

for a Borel set $E \subset \delta-S$. By substituting the class

$$
Q_{S}=\left\{u \in C^{2}(R) \mid \Delta u=l_{E}, E \subset \delta-S\right\}
$$

for $Q$ in $\$ 1$, we can deduce similar but more precise results than those in $\$ \S 3$ and 4 . An analysis of the classes $O_{Q_{s} X}(X=P, B, D, C)$ and, from a somewhat more general viewpoint, the classification problem for $O_{W X Y_{\Delta}}(X, Y=B, C)$ are important further topics; here $O_{W X Y_{\Delta}}$ is the class of Riemannian manifolds $R$ on which $W X Y_{\Delta}(R)$ reduces to constants.

\section{REFERENCES}

1. S. Bergman and M. Schiffer, Kernel functions and elliptic differential equations in mathematical physics, Academic Press, New York, 1953. MR 14, 876.

2. C. Constantinescu and A. Cornea, Ideale Ränder Riemannscher Flächen, Ergebnisse der Mathematik und ihrer Grenzgebiete, Band 32, Springer-Verlag, Berlin, 1963. MR $28 \# 3151$.

3. Y. K. Kwon, L. Sario and B. Walsh, Behavior of biharmonic functions on Wiener's and Royden's compactifications, Ann. Inst. Fourier (Grenoble) (to appear).

4. M. Nakai and L. Sario, Quasiharmonic classification of Riemannian manifolds, Proc. Amer. Math. Soc. (to appear).

5. L. Sario and M. Nakai, Classification theory of Riemann surfaces, Die Grundlehren der math. Wissenschaften, Band 164, Springer-Verlag, New York, 1970.

University of California, Los Angeles, California 90024 